\title{
Is preputioplasty effective and acceptable?
}

\author{
N J Barber BSc FRCS(Urol) \\ B Chappell MRCs ${ }^{1}$ \\ P G Carter MD FRCS(Urol $)^{2}$ \\ J P Britton MD FRCS ${ }^{2}$
}

J R Soc Med 2003;96:452-453

\section{SUMMARY}

Foreskin complaints in childhood, if not manageable conservatively, are usually treated by circumcision. A less radical surgical option, when balanitis xerotica obliterans is absent, is preputioplasty. We sent questionnaires to the parents of 23 boys who had had this procedure and 22 replied.

Mean interval since operation was 20 months (range 3-36). The main indications for surgery had been irretractable foreskin in 9, recurrent balanoposthitis in 10 and ballooning on voiding in $\mathbf{3}$ and the operation had dealt successfully with these in 7, 7, and 3, respectively. In all but one case the parents were satisfied with the cosmetic result. However, in 8 cases $(36 \%)$ the parents said they would have preferred circumcision and 3 of the boys had been listed for further surgery.

Preputioplasty is a satisfactory alternative to circumcision in selected cases.

\section{INTRODUCTION}

Foreskin complaints that commonly cause children or their parents to seek medical advice are pain, recurrent balanoposthitis and inability to retract the prepuce. These conditions, which often relate to phimosis, will sometimes respond to conservative management but many boys are treated by circumcision. Parents often press hard for this operation. However the morbidity of circumcision is well recognized and in some cases the loss of the foreskin will be regretted later. A quicker, simpler and less radical procedure is preputioplasty. We sent questionnaires to the parents of 23 boys who had had this operation.

\section{METHODS}

23 boys (mean age 5 years; range 1-10) underwent preputioplasty as a day case under the care of two consultant urologists in the course of 3 years. The procedure was offered to those who had a problematic irretractable prepuce with a clear tight band and no evidence of balanitis xerotica obliterans (lichen planus et atrophicus). Under general anaesthesia, a dorsal longitudinal incision is made through the tight preputial band. After haemostasis, the wound is closed horizontally with a $4 / 0$ Vicryl Rapide suture (Figure 1). Parents were asked to begin retraction of the foreskin the next day, placing $2 \%$ lidocaine gel behind it, and to continue for three days. The questionnaire (available from the authors) asked parents to

Departments of Urology, King's College Hospital, London; ${ }^{1}$ Royal Sussex County Hospital, Brighton; ${ }^{2}$ St Richard's Hospital, Chichester, UK

Correspondence to: Mr N J Barber, 1 Grove Mews, Hammersmith, London W6 7HS, UK address nine questions concerning the outcome and their satisfaction.

\section{RESULTS}

Replies were received from 22 of the 23 sets of parents at a mean interval of 20 months (range 3-36) from operation. There had been no postoperative complications - and all but 2 boys had been back to normal activities within ten days. In terms of the main indications for operation, the procedure was rated successful as follows: irretractable foreskin 7 of 9 , recurrent balanitis 7 of 10, ballooning on voiding 3 of 3 . Of the whole group, 17 boys (77\%) now had a retractable prepuce. In all but 1 case, the parents were happy with the cosmetic result. However, in 8 cases
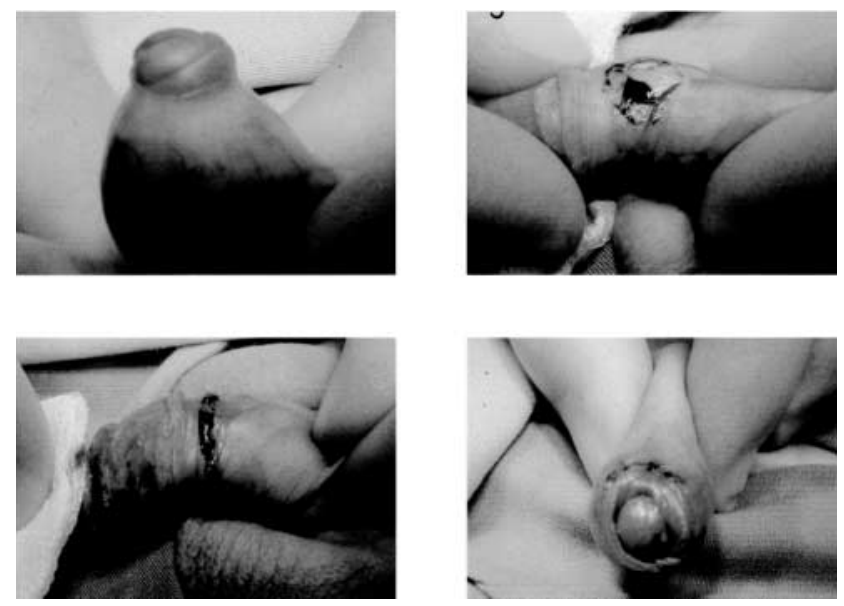
(36\%) the parents would have preferred circumcision and 3 boys were to have further surgery.

\section{DISCUSSION}

There are good reasons for avoiding circumcision if possible. Though one of the most commonly performed operations worldwide it still carries substantial morbidity including haemorrhage, meatal ulceration and stricture. In addition there is some evidence of late adverse effects on sexual function. The foreskin is suspected to be important in terms not only of male sensation but also of female enjoyment of intercourse. ${ }^{1-3}$

One of the main reasons for circumcision is an irretractable prepuce, and parents commonly press for the operation. However, in many instances the 'phimosis' is physiological, and the adhesions can be expected to break down spontaneously; by the age of 17 , only $1 \%$ remain irretractable. ${ }^{4}$ Where operation is required, the lesser procedure of preputioplasty (also known as preputial plasty or prepuce plasty), though still little used, has distinct merits; and experience with the present series confirms its low rate of postoperative complications and its good results in terms of preputial retractability and cosmetic outcomes. ${ }^{5-9}$ Apart from the single dorsal incision used in this series, techniques for preputioplasty include two lateral incisions and a 'triple incision plasty' with three incisions across the fibrotic bands either longitudinally or obliquely; the oblique method is claimed to carry less risk of a dog-ear effect. Our success rate in preventing recurrence of balanoposthitis was disappointing (70\%), but we do not know the extent to which parents applied the recom- mended care: regular postoperative retraction is important to prevent early readhesion. Another disappointment was the proportion of parents (one-third) who would have preferred circumcision from the outset.

A disadvantage of our retrospective approach is that we cannot draw conclusions about selection criteria and their validity. However, coupled with previously reported results, experience with this series leads us to believe that, where there is a tight band of tissue, preputioplasty should be on the list of therapeutic options. Where generalized atrophic and sclerotic changes are present, circumcision remains the treatment of choice.

\section{REFERENCES}

1 Laumann EO, Masi CM, Zuckerman EW. Circumcision in the United States: prevalence, prophylactic effects and sexual practice. JAMA 1997;227:1052-7

2 Hammond T (National Organisation to Halt the Abuse and Routine Mutilation of Males). A preliminary poll of men circumcised in infancy or childhood. BJU Int 1999;83(suppl. 1):85-92

3 O'Hara K, O'Hara J. The effect of male circumcision on the sexual enjoyment of the female partner. BJU Int 1999;83(suppl. 1):79-84

4 Oster J. Further fate of the foreskin. Arch Dis Child 1968;43:200-3

5 Lane TM, South LM. Lateral preputioplasty for phimosis. J R Coll Surg Edinb 1999;44:310-12

6 Cuckow PM, Rix G, Mouriquand PDE. Preputial plasty: a good alternative to circumcision. J Pediatr Surg 1994;29:561-3

7 de Castella H. Prepuceplasty: an alternative to circumcision. Ann R Coll Surg Engl 1994;76:257-8

8 Holmlund DEW. Dorsal incision of the prepuce and skin closure with dexon in patients with phimosis. Scand J Urol Nephrol 1973;7:97-9

9 Wahlin N. 'Triple incision plasty'. A convenient procedure for preputial relief. Scand J Urol Nephrol 1992;26:107-10 